\title{
研究論文
}

\section{A r ray Spectrometric Method for the Measurement on Fall-out Contaminated Samples}

\author{
フォールアウト試料の $\gamma$ 線スペクトロメトリー \\ By Mikio YAMASHITA* and Hironobu WATANABE*
}

\begin{abstract}
From the standpoint of feasible application to the measurement on the samples cotaminated by fall-out, a practical $\gamma$ ray spectrometric method was developed. It contains a statistical method for analyzing the spectra and an experimental method for estimating a relative photopeak efficiency vs. energy.

In this method, only one standard of certain energy is needed and subsequent absolute mecisurement for other nuclides can be attained with a knowledge of a relative photopeak efficiency.
\end{abstract}

\section{INTRODUCTION}

$\gamma$ ray spectrometric methods for quantitative analysis of fall-out samples such as rain water, vegetables, soil and the like have been widely employed for the usefulness in rapid determination of the $\gamma$ ray emitting nuclides. And these techniques have been greatly improved by the recent development of multichannel analyzers which would offer more rapid data processing by means of computer systems.

However, it still remains as a difficult problem to precisely analyze the $\gamma$ ray spectra, especially in case of complicated ones.

In case of mixed spectra containing several $\gamma$ ray energies, the spectrum-stripping method has been frequently used in order to derive the photopeak area from a single $\gamma$ ray of interest $^{(1) \sim(4)}$. This is based on repetitive subtraction of each component spectrum, starting with that of the highest enenrgy. This method is, unfortunately, tedious and requires highly skillful techniques. On the other hand, since multi-channel analyzers have been available, statistical methods or similar kind of data analysis have been developed for analysis of $\gamma$ ray spectra ${ }^{(5) \sim(9)}$. The authors actually applied the method of least squeres to the determination of trace amount of ${ }^{137} \mathrm{Cs}$ in soil from the $\gamma$ ray spectra with satisfactory results ${ }^{\cos (11)}$. In fact, the latter mothod should be preferable especially for routine analysis at low counting rates.

The quantitative determination of a nuclide of interest from a $\gamma$ ray spectrum requires the accurate measurement of the area under the photopeak or the peak height. The absolute value of disintegration rate can be obtained by comparing it with that due to a standard or by absolute calculation ${ }^{(12)}$.

The practical application of the method by absolute calculation is described somewhere ${ }^{(13) \sim(15)}$.

However, since the calculation of a photopeak efficiency with consideration of geometry and status of the sample is quite involved, this method often faces difficulties in ordinary use, when it comes to measurement of fall-out contaminated samples which are not necessarily mounted in a simple form.

If standards of individual nuclides present in a $\gamma$ ray spectrum are easily available with the same geometrical condition as that of the

* 山下幹雄, 渡辺博信。National Inst. Radiological Sci.（放射線医学総合研究所) 
sample, there is no problem for the quantitative analysis, but unfortunately this is in many cases beyond a reach of common laboratories.

Therefore, it is the purpose of this paper to describe a practical method for quantitative determination of radionuclides from $\gamma$ ray spectra obtained by a multi-channel analyzer. The base of this method is to analyze the spectra by a statistical method and to draw up a curve of relative photopeak efficiency vs, energy by a possibly suitable method that could be carried out at most laboratories.

\section{Analysis of Spectrum}

\section{Photopeak area}

The pulse height spectrum of a single monoenergetic $\gamma$ ray by a sodium iodide crystal consists mainly of a Compton continium resulting from scattering within the crystal and the surrounding shielding, and a photopeak resulting mainly from the photoelectric effect within the crystal. The latter is generally almost indistinguishable from Gaussian distribution, especially at the high energy side of the peak.

Then, $S_{\mathfrak{t}}$ being the count rate in channel $i$, a photopeak may be expressed as follows,

$$
S_{i}=H \exp \left(-h^{2} x_{i}^{2}\right) \text {, }
$$

where $H$ is the peak height, $h$ a constant and $x_{i}$ is the distance from the original point being at the center of the peak.

The most probable value of $H$ can be obtained by minimizing $R$ of the following equation.

$$
R=\Sigma\left\{S_{i}-H \exp \left(-h^{2} x_{i}^{2}\right)\right\}^{2}
$$

Thus $H$ is expressed as follows,

$$
H=\frac{\sum a_{i} S_{t}}{\sum a_{i}^{2}} .
$$

where $\quad a_{i}=\exp \left(-h^{2} x_{i}^{2}\right)$.

The value of $h^{2}$ could be easily obtained by using a probability scale paper where half the part of the photopeak at the high energy side is preferably used for the reason mentioned previously.

Fig. 1 shows an example to obtain the value of $h^{2}$ in case of a ${ }^{137} \mathrm{Cs}$ photopeak and the variation of $h^{2} v s$. energy obtained by a $13 / 4^{\prime \prime}$ $\phi \times 2^{\prime \prime} \mathrm{NaI}(\mathrm{Tl})$ crystal is examplified in Fig. 2 .

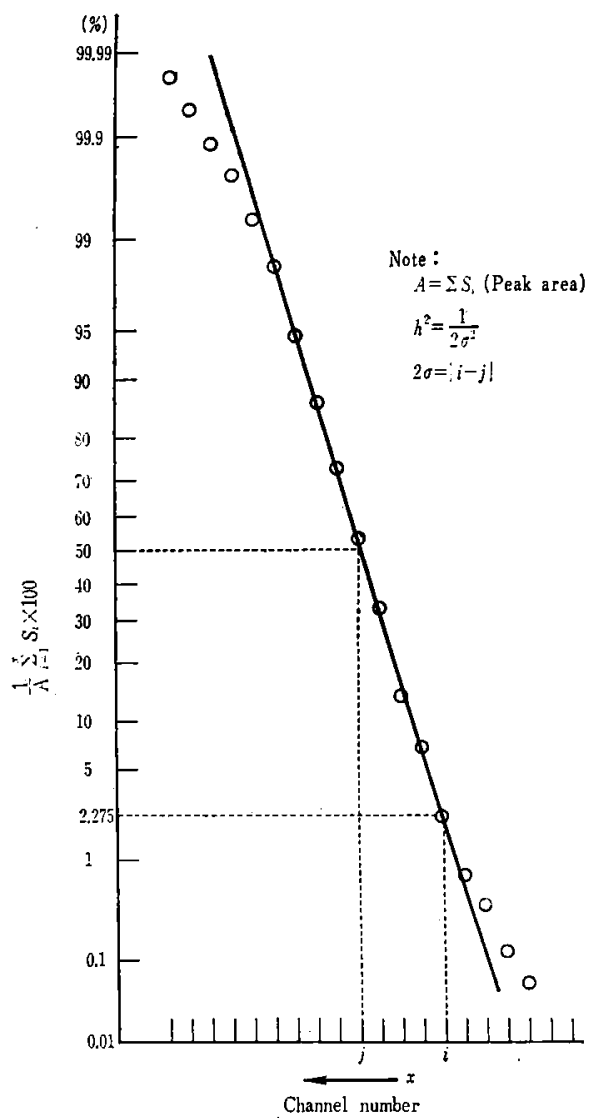

Fig. 1 Estimation of the value of $h^{2}$ of Gaussian distribution by means of probability paper

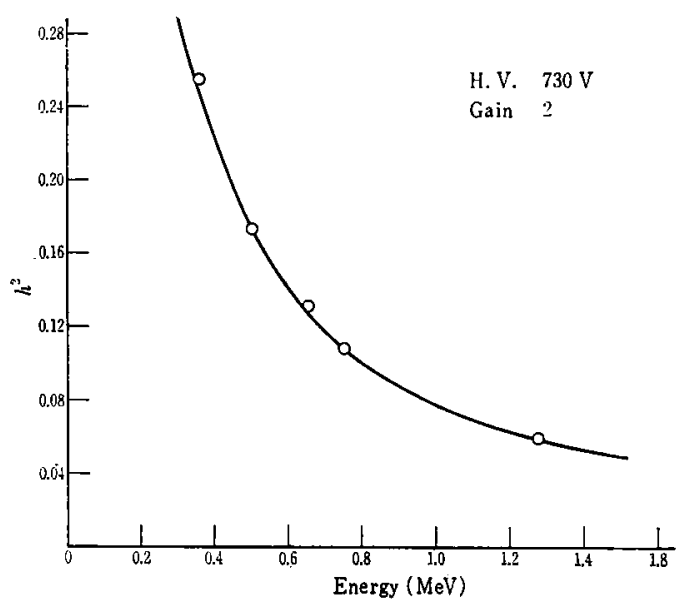

Fig. 2 The variation of the value of $h^{2}$ of Gaussian distribution with energy 
The photopeak area $A$ can be given by

$$
A=C H W \text {, }
$$

where $C$ is a constant and theoretically equivalent to 1.07 . $W$, the width at half the height of the Gaussian distribution is mathematically given by

$$
W=\frac{1.665}{h} \text {. }
$$

The variation of the half width of the peak with $\gamma$ ray energy is well approximated by ${ }^{(16)(17)}$

$$
W=K E^{\alpha},
$$

where $\alpha$ is a constant. Shown in Fig. 3 is the half width $v s$. energy obtained from Eq. (5) and Fig. 2.

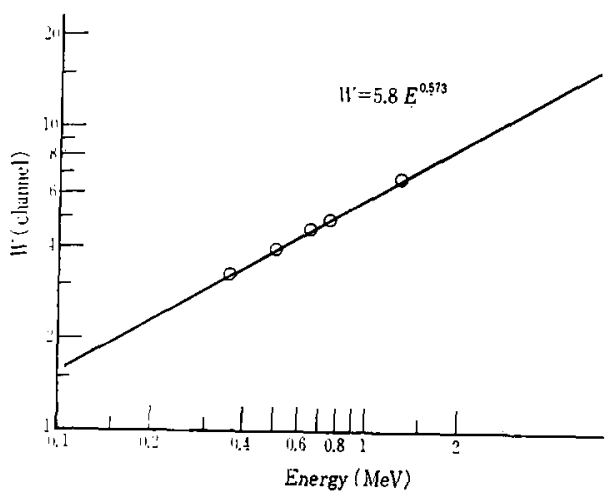

Fig. 3 The variation of the peak half width with energy

\section{Statistical analysis of spectrum}

In a statistical treatment of a $\gamma$ ray spectrum, the narrow region around a photopeak of interest could be approximated as follows,

$$
S_{t} \simeq H \exp \left(-h^{2} x_{i}^{2}\right)+g\left(x_{i}\right),
$$

where $S_{i}$ is the count rate in channel $i, H$ being the photopeak height with a Gaussian distribution and $g(x)$ indicates the contribution from other $\gamma$ rays present in the spectrum to the region of interest. $g(x)$ might be expressed simply by a straight line or more precisely by a parabola or more complex functions.

Taking a parabola for $g(x)$, Eq. (7) becomes

$$
S_{i}=H \exp \left(-h^{2} x_{i}^{2}\right)+A+B x_{i}+C x_{i}^{2},
$$

where $A, B$ and $C$ are constants.

When one may consider the deviation of count rate $\left(S_{t}\right)$ observed, application of the least squares method yields the following weighted normal equation.

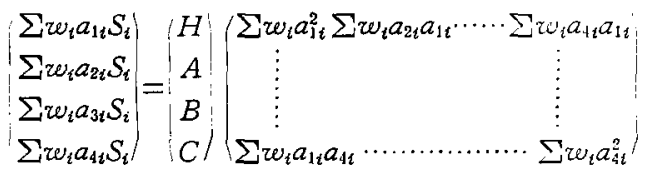

(9)

where $w_{i}=\sigma_{2}^{0} / \sigma^{2}, a_{1 t}=\exp \left(-h^{2} x_{i}^{2}\right), a_{2 t}=1, a_{3 t}=x_{i}$, $a_{4 i}=x_{i}^{2}, \sigma_{0}$ being an arbitrary constant and $\sigma$ is the standard deviation of count rate $S_{v}$. However it should be mentioned that since the weight factor $w_{t}$ in Eq. (9) is unlikely to affect greatly the result, it might be omitted in the ordinary cases.

From Eq. (9), $H$ is given by

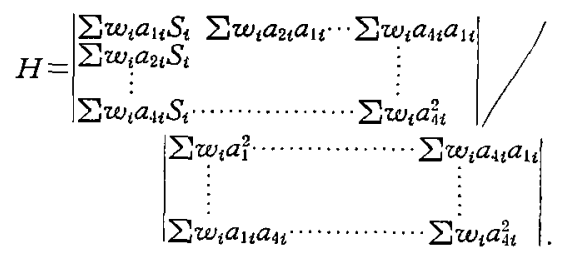

The standard deviation $\sigma_{H}$ of $H$ derived through the above calculation is mathematically given as follows,

$$
\sigma_{H}=\sigma_{0} \sqrt{\frac{I}{w_{H}}},
$$

where $w_{H}$ is a relative weight of the value of $H$, the reciprocal of which is expressed as the solution of $H_{0}$ in the following equation ${ }^{(18)}$

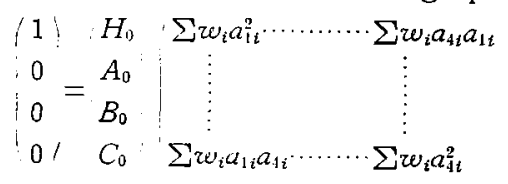

\section{Conversion to Absolute Disintegration RATE}

The photopeak efficiency (relative magnitude of the photopeak area)generally decreases with an increase of $\gamma$ ray's energy. If both a knowledge of a relative photopeak efficiency vs. energy and a standard sample of energy $E_{s}$ with the same condition as that of the samples to be measured are available, one can convert the peak area of a certain energy to the absolute disintegration rate as described below.

$$
D_{i}=D_{s} A_{A_{s}}^{A_{i}} \cdot \frac{\varepsilon_{E_{s}}}{\varepsilon_{E_{t}}} \cdot \frac{\eta_{s}}{\eta_{i}},
$$


where $D:$ the disintegration rate

$A$ : the photopeak area

$\varepsilon$ : the relative photopeak efficiency of $\gamma$ ray energy $E$

$\eta$ : the abundance of the $\gamma$ ray of interest

and the suffixes $i$ and $s$ denote the sample and the standard respectively.

\section{Relative Photopeak EFFiciency}

By using an isotope that emits simultaneously more than two $\gamma$ rays with known $\gamma$ abundance, one can estimate a relative photopeak efficiency corresponding to each $\gamma$ ray. However, since the accuracy greatly depends upon the determination of the peak area, it should be preferable to make use of the nuclides of simple decay scheme.

On the assumption that photopeak efficiency should decrease monotonously with an increase of energy, one can approximately unite the groups of relative photopeak efficiency which are independently obtained by using several isotopes.

Suppose that we have now got the ratio, $k_{1}$ of the photopeak efficiency at energies of $E_{t}$ and $E_{j}$ emitted from an isotope with the known $\gamma$ abundance, and $k_{2}$, the ratio of the photopeak efficiency at energies of $E_{m}$ and $E_{n}$ from another.

When $k_{1}$ and $k_{2}$ are reasonably correlated, the product $S$ of the area of $\triangle P_{1} P_{2} P_{3}$ and that of $\Delta P_{2} P_{3} P_{4}$ in Fig. 4 should be minimized.

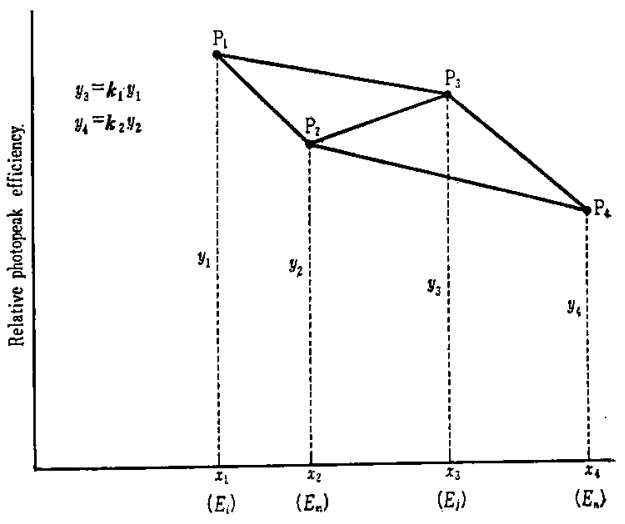

Fig. 4 Unification of two separate groups of relative photopeak efficiency

$$
\begin{aligned}
S=\frac{1}{4} & \left\{y_{1}\left(x_{3}-x_{2}\right)+y_{2}\left(x_{1}-x_{3}\right)+y_{3}\left(x_{2}-x_{1}\right)\right\} \\
& \left\{y_{2}\left(x_{4}-x_{3}\right)+y_{3}\left(x_{2}-x_{4}\right)+y_{4}\left(x_{3}-x_{2}\right)\right\}
\end{aligned}
$$

Thus the value of $y_{2}$ or $y_{4}$ correlated to $y_{1}$ or $y_{3}$ in Fig. 4 can be approximated by

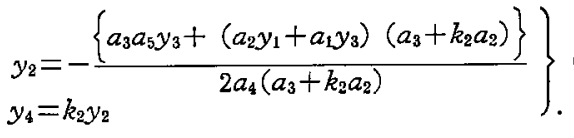

where $a_{1}=x_{2}-x_{1}, a_{2}=x_{3}-x_{2}, a_{3}=x_{4}-x_{3}$,

$$
a_{4}=x_{1}-x_{3}, a_{5}=x_{2}-x_{4} \text {. }
$$

As the same procedures are extended oneafter another by using different isotopes suitable for the purpose, one can draw a curve of relative photopeak efficiency by interpolation.

Fig. 5 shows a relative photopeak efficiency vs. energy with a $13 / 4^{\prime \prime} \phi \times 2^{\prime \prime} \mathrm{NaI}(\mathrm{Tl})$ crystal, obtained by using point sources of ${ }^{144} \mathrm{Ce},{ }^{131} \mathrm{I}$. and ${ }^{22} \mathrm{Na}$, and the description of these nuclides. are shown in Table $1^{(19)(20)}$.

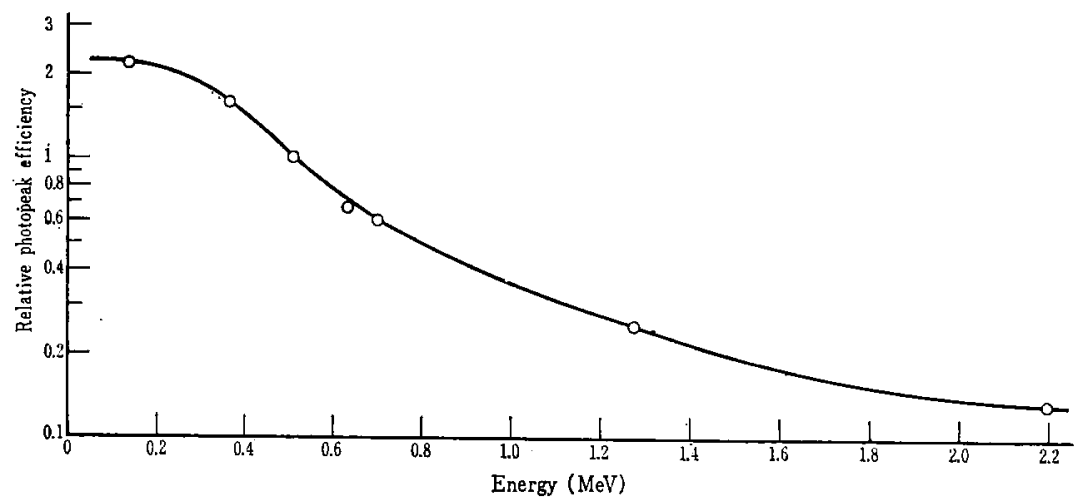

Fig. 5 Estimated relative photopeak efficiency with a $13 / 4 " \phi \times 2^{\prime \prime} \mathrm{NaI}(\mathrm{Tl})$ crystal 
Table 1 The isotopes used for estimating a relative photopeak efficiency

\begin{tabular}{c|c}
\hline Nuclide & $\begin{array}{c}\gamma \text { ray's energy of interest } \\
(\mathrm{MeV})\end{array}$ \\
\hline${ }^{22} \mathrm{Na}$ & $0.51(\sim 200 \%)$ \\
$(2.6 \mathrm{y})$ & $1.276(\sim 100 \%)$ \\
\hline${ }^{131} \mathrm{I}$ & $0.364(80 \%)$ \\
$(8.1 \mathrm{~d})$ & $0.637(9 \%)$ \\
\hline & $0.133(17.2 \%)$ \\
${ }^{144} \mathrm{Ce}$ & $0.694(1.6 \%)$ \\
$(285 \mathrm{~d})$ & $2.18(0.8 \%)$ \\
\hline
\end{tabular}

As far as the measurement of fall-out contaminated sample is concerned, the energy range of about 0.1 to $2 \mathrm{MeV}$ in the curve can fully serve the purpose.

\section{Discussion}

In a accurate evaluation of the photopeak area, there are several influences to be taken into account such as Compton edge, backscattering, sum effect, escape peak, etc (12)(21).

Especially the low energy portion of the spectrum is more likely to be in error by such influences as Bremsstrahlung, detector noise and other factors.

However, in the ordinary application of the statistical method described above to the spectrum of fall-out samples where ${ }^{{ }^{141}} \mathrm{Ce}$, ${ }^{144} \mathrm{Ce},{ }^{103} \mathrm{Ru},{ }^{106} \mathrm{Ru}$ and $\mathrm{Zr}+{ }^{95} \mathrm{Nb}$ were dominant components, the magnitude of error did not exceed $10 \%$.

Although unfortunately the authors have not got enough knowledge about to what extent a relative photopeak efficiency vs, energy be influenced by the geometrical conditions of the sample and the detector, it is unlikely that a relative photopeak efficiency would significantly vary by a little change of the geometrical conditions ${ }^{(22)}$.

In case of cylindrical samples where the self-absorption effect might play an important part, the circumstance would be different and the authors are going to discuss this matter at another opportunity.
The authors wish to express thanks to Mr. S. Abe and Mr. Y. Oguchi for their helpful discussion on the statistical treatment of the spectra. Thanks are also due to Dr. M. Okano, Dr. R. Ichikawa and Mr. T. A. Iinuma for their helpful criticism of the manuscript.

(Received February 8, 1963)

- REFERENCES -

(1) R. E. Conally: Anal. Chem., 28, No. 12, 1847 (1956).

(2) W. LEE: ibid., 31, No. 5, 800 (1959).

(3) P. F. Gustafson: Science, 127, 1240 (1958).

(4) M. GeIsler, et al: : Atomkernenergie, 7, No. 1, No. 21 (1962).

(5) L. Salmon: Nucl. Instr. Methods, 14, No. 2, 193. (1961).

(6) P. McWilliams, et al.: Rev. Sci. Instr, 33, No. 1, 70 (1962).

(7) H. M. ChILders: ibid., 30, No. 9, 810 (1959).

(8) TID-7594, (1960).

(9) R. L. Health: Nucleonics, 20, No. 5, 67 (1962).

(10) H. WatanaBe, et al.: Ann. Rept. National Inst. Radiological Sci., 129 (1960).

(11) H. WATANABE, et al.: The $3 \mathrm{rd}$ Meeting of the Japan Radiation Research Society, (Aug. 1961).

(12) C. E. Crouthamel (Ed.): "Applied Gammaray Spectrometry", (1960), Pergamon Press.

(13) IDO-16408, (1957).

(14) N. H. LAZAR: IRE, Trans. Nucl. Science, 138 (1958).

(15) TID-7591, (1959).

(16) G. G. KeLly, et al.: Nucleonics, 14, No.4, 53 (1956).

(17) R. E. Conally, et al.: Anal. Chem., 25, 1095 (1953).

(18) S. Numakura: "Sokuteichi Keisan-ho", (1959), Morioka Publ. Co., Tokyo.

(19) D. Strominger, et al.: Rev. Modern Phys., 30, No. 2, Part 2, (1958).

(20) H. Houtermans, et al.: Intern. J. Appl. Radiation and Isotopes, 13, 137 (1962).

(21) K. Siegbahn (Ed.): "Beta- and Gamma-ray Spectroscopy", (1955), North-Holland Publ. Co., Amsterdam.

22) W. F. MILLER, et al.: ANL-5902, (1958). 